\title{
SPLENIC ABSCESS: REVIEW ARTICLE
}

Sanjay M. Datey ${ }^{1}$, Neelam Charles ${ }^{2}$, Himanshu Patidar ${ }^{3}$, Shibanshu Bandil ${ }^{4}$, Shaurya Bajpai ${ }^{5}$, Mohan Gadodia6

\section{HOW TO CITE THIS ARTICLE:}

Sanjay M. Datey, Neelam Charles, Himanshu Patidar, Shibanshu Bandil, Shaurya Bajpai, Mohan Gadodia. "Splenic Abscess: Review Article". Journal of Evolution of Medical and Dental Sciences 2015; Vol. 4, Issue 32, April 20; Page: 5569-5576, DOI: 10.14260/jemds/2015/814

ABSTRACT: Case report of splenic abscess in a 35 year old male is presented with review of the literature. The patient was admitted with left sided lower chest pain and fever without any significant abnormality on physical examination and haematological investigations. On imaging studies a large splenic abscess was diagnosed which was initially treated by broad spectrum antibiotics. Aspiration under sonographic control was unsuccessful due to thick pus and debris in the abscess. Open splenectomy was done with a complete recovery of the patient. Splenic abscess is an infrequent clinical entity and carries a very high mortality in the untreated cases. Pathognomic clinical features of left hypochondrial pain, splenomegaly, fever and leucocytosis are present in only one-third of the cases. Diagnosis is mostly done by imaging, either Abdominal Sonography or CT Scan. So far no prospective study has been done to rationalize the management, probably due to paucity of cases. Antibiotics alone or with percutaneous drainage under imaging control are successful in many cases. Failed aspirations and splenic abscesses with concomitant pathologies need splenectomy. Splenectomy and complete removal of the source of the infection is still the mainstay of treatment. Detailed review of the available literature of the clinical problem and various methods of treatment of splenic abscesses are discussed in the article.

KEYWORDS: Splenic Abscess, Percutaneous drainage of splenic abscess, Splenectomy.

INTRODUCTION: Splenic abscess is an infrequent clinical entity. International literature has less than 600 reported cases so far.(1) Splenic abscesses have a very high mortality in untreated cases. Pathognomic clinical features of left hypochondrial pain, fever, splenomegaly and leucocytosis are present in only one-third of the cases. Diagnosis is mostly done by imaging i. e., Sonography or CT Scan. Various treatment modalities like aspiration under imaging control with appropriate antibiotics and splenectomy by open or laparoscopic approach have been described. So far no prospective study has been done to rationalize the management probably due to the paucity of cases.

CASE REPORT: Thirty five year old male was admitted with history of pain in the left lower chest with spikes of fever for the eight previous days. Intensity of the throbbing pain was increasing over the period of time. Pain was localised in left lower back region and in left hypochondrium without any aggravating or relieving factors. Moderate fever characterized by spikes was present. Rest of the history did not reveal any other significant information. General physical examination did not reveal any abnormality except for tenderness on percussion over the posterior aspect of lower rib cage.

Haematotological and biochemical parameters were within normal range. Renal and hepatic functions were normal and the patient was seronegative for HIV and Hepatitis. X-ray of the chest did not show any abnormality. Ultrasonography of the abdomen revealed perisplenic collection with moderate splenomegaly. Other abdominal organs were normal on sonography. Contrast enhanced computerised tomography (CECT) was instrumental in diagnosis of splenic abscess. 
CECT of the abdomen reported a large subcapsular collection with markedly non enhancing areas suggestive either of splenic infarct with liquefactive necrosis with subcapsular haematoma or splenic rupture with liquefactive subcapsular and intraparenchymal haematoma. Most probable diagnosis was that of a splenic abscess. Broad spectrum antibiotics with the supportive treatment was started and aspiration under ultrasound control was planned. Abscess cavity was full of debris and that is why aspiration failed. Condition of the patient deteriorated in spite of antibiotics and a decision for abdominal exploration with splenectomy was taken. Exploratory laparatomy was done by upper midline incision. We noticed a moderately enlarged spleen, a perisplenic haematoma, dense omental adhesions with hilum of spleen and adhesions to peritoneal and diaphragmatic surfaces of spleen. Splenectomy performed and a thorough peritoneal lavage was given. No spillage or no other intraabdominal pathology was noted. Postoperative recovery was uneventful and the patient was discharged from the hospital on 12th postoperative day. The histopathological examination revealed a large splenic abscess with debris filling almost two-third of the spleen. No evidence of any other pathology in the spleen was found.

DISCUSSION AND REVIEW OF LITERATURE: Splenic abscess is an uncommon disease and review of literature in this matter revealed that no prospective study has been conducted so far.

INCIDENCE: Splenic abscesses are uncommon and all cases are not registered, hence true incidence is difficult to know. Published autopsy statistics states the incidence to be $0.05 \%$ to $0.7 \%$. Incidence varies according to the geographical areas and the population under the study. A wide variation in the causative organism, demographic pattern and clinical presentation is observed. Patients of both sexes are affected equally and middle to old aged people are common sufferers.(2)

ETIOLOGY: Current understanding of the exact etiology of a splenic abscess is limited. Commonest predisposing factors are bacterial endocarditis, splenic trauma and diabetes mellitus. Splenic abscesses are associated with following comorbidities.

Bacterial Endocarditis.

Diabetes mellitus.

Acquired immunodefeciency syndrome (AIDS) and HIV infection.

Immunosuppressive states.

Malignancies.

Conservatively treated splenic trauma.

Formation of an abscess in the spleen can be explained in the following ways:

1. SEPTIC EMBOLISATION: Septic emboli reach the spleen through blood stream and lodge in the parenchyma. Vegetations from subacute bacterial endocarditis is the commonest cause of splenic abscess by this method. Central line sepsis, bacterimia and fungimia in immunocompromised patients on chemotherapy and AIDS can lead to a splenic abscess in this way.

2. ALTERED SPLENIC ARCHITECTURE DUE TO TRAUMA: Conservatively treated splenic trauma patients can get an abscess of spleen due to infection of the haematoma. Splenic infarcts due to leukamic deposits, sickle cells disease or vasculitis can also be a starting point of an abscess.

3. CONTAGIOUS SPREAD FROM SURROUNDING PATHOLOGIES: Necrotising pancreatitis with abscess of lesser sac can spread to spleen. Other cause of contagious spread can be gastric or colonic perforations. 
4. MICROABSESSES OF SPLEEN IN HIV-INFECTED: Multiple microabscesses of spleen were diagnosed by high resolution ultrasound (7.5Mhz) in patients of AIDS in a study.(3) This finding suggests that splenic microabscesses may be a frequent condition in HIV-infected patients with prolonged fever which goes unnoticed in conventional ultrasound by 3.5 Mhz probe.

PATHOLOGY AND MICROBIOLOGY: Spleen is an uncommon site of infection and abscess formation. The intrinsic immunological function may give relative protection against suppurative infections. Focus of infection in an immunocompromised state or disturbed architecture of spleen with a large load of septic material like an embolus from subacute bacterial endocarditis leads to abscess. Single or multiple foci can be found which in turn lead to single or multiple abscesses. Splenic abscess es are polymicrobial in $50 \%$ of cases. Wide spectrum of strains of organism are isolated on culture of pus from a splenic abscess. Gram positive cocci namely Streptococci, Staphylococci and Enterococci are predominantly seen in most of the cases. Gram negative bacilli like the Escherichia Coli, Klebisiella Pneumonie and Pseudomonas Aeruginosa are occasionally predominant.(4) Salmonella strains can be seen as the offenders in endemic areas of Typhoid fever.(5,6,7) Isolated tuberculous abscess or involvment of spleen in miliary tuberculosis can be seen. $(8,9,10)$ Contagious spread from colonic perforations can have infection due to anaerobes like Bacteroides, Fusobacterium, Clostridium, and fungi like Candida and Aspergillus.(11,12,13) Meliodosis is reported as the commonest cause in countries like Taiwan and Singapore.(14,15)

CLINICAL PRESENTATION: Splenic abscess is an uncommonly seen condition and thus remains a diagnostic challenge. Classical triad of left hypochondrial pain, fever and splenomegaly is present in only one third of the patients of splenic abscess. Fever can be moderate and continuous or intermittent. Sometimes fever can be absent. Left hypochondrial dull aching is due to perisplenitis. Presence of persistent pain and tenderness can be an indication of impending rupture of abscess. Splenomegaly is not a constant feature and it is found only in one third of cases. In a retrospective study of 75 cases, splenomegaly was found only in 41 cases.(2) Left pleuritic pain and reactionary left pleural effusion can be seen. Irritation of under surface of diaphragm can lead to left soulder tip pain or hiccoughs. Many patients do not show any of these features except fever and leucocytosis, and are diagnosed by ultrasound or CT Scan.

INVESTIGATIONS: Haematological investigations show leucocytosis with rest of the parameters within normal range. $\mathrm{C}$ reactive proteins are raised. Biochemical parameters are not affected by splenic abscess. Blood culture and culture-sensitivity of pus aspirated from abscess are mandatory for appropriate antibiotic use. Atypical infections like tuberculosis, fungi like candida and aspergillus and HIV should always be kept in mind while investigating a case.

IMAGING STUDIES: Chest X-ray reveals nonspecific changes due to a septic focus in the region like elevated left hemidiaphragm and left pleural effusion.(2) An abnormal finding on plain x-ray of abdomen could be an abnormal soft tissue shadow or gas collection with a fluid level in the left hypchondrial region. Imaging studies, Ultrasound and CT scan have eminent role in the diagnosis and management of splenic abscesses. Ultrasonography gives information about the number, size and location of an abscess in the spleen. It also tells us whether an abscess is unilocular or multilocular. 
CT scan is reproducible and not observer dependent and also gives better information about the concommitant pathologies in surrounding viscera. MRI and Radioisotopic studies can also be done but have alimited role.

ULTRASONOGRAPHY: Most of the abscesses of spleen are diagnosed by Ultrasonography only. It is cost effective, noninvasive and readily available at the bedside round the clock however the evaluation is nonspecific and operator dependent. USG can help in the diagnosis and can help in obtaining pus for culture and sensitivity for starting appropriate antibiotics. Splenic abscess can be missed in presence of concomitant lesser sac collections or subdiaphragmatic abscess.

CT SCAN: CT Scan is the standard criteria for dignosis of a splenic abscess. Reported sensitivity of CTScan for this purpose approaches $100 \%$. The characteristic image of a splenic abscess reveals low density lesions that fail to enhance after intravenous contrast. CTScanning best delineates the size, topography and access route to the spleen and surrounding structures.(16) CT guided drainage can be performed during examination or afterwards by deciding the route.

MANAGEMENT OF SPLENIC ABSCESS: Options for the management of a splenic abscess are;

1. Broad spectrum antibiotics to start with and tailored according to senstivity.

2. Percutaneous drainage either by aspiration or drainage by retaining a catheter in abscess cavity.

3. Open or laparascopic splenectomy.

4. Open drainage of abscess by laparotomy.

ANTIBIOTICS: As a first line therapy when awaiting results of culture and all other investigations. Patient is moribund and not fit for anasthesia and surgery and aspiration has not been successful. Multiloculated or multiseptate abscessesnot amenable to percutaneous drainage.

Broad spectrum antibiotics are started to cover polymicrobial flora. Absence of fever, improvement in the general condition of the patient are indicators of a good clinical response. Further assesment can be done by review sonography which will show a decrease in size of abscess. Failure of antibiotic treatment is indicated by deteriration in the condition of patient with increasing spikes of fever, marked tachycardia, localized guarding in the left hypochondrium and signs of overwhelming peritonitis. Ultrasonography may show increase in size of the abscess or multiple abscesses with or without involvement of adjacent organs. Decision to intervene either by surgery to remove the spleen or drainage of pus is critical and has to be reached entirely on clinical grounds as a delay can lead to septicemia and multiple organ failure.

PERCUTANEOUS DRAINAGE: Percutaneous drainage can be done under USG control when the abscess is near the parietal wall and preferably unilocular. Multilocular or centrally located abscess is not suitable for percutaneous drainage. In a multilocular abscess pus can obtained for culture sensitivity test to select an appropriate antibiotics. Percutaneous drainage is better tolerated and acceped in moribund patients. Preservation of the spleen is the biggest advantage of this method. However percutaneous drainage has some limitations as it cannot be used in multifocal and can be dangerous on inaccessible abscesses. Septate collections and necrotic debris cannot be removed 
completely, leaving behind the infective focus. Percutanoeous drainage is not as common in spleen as in other anatomical sites, probably because of fear of uncontrollable bleeding. With increasing experience US guided aspiration is becoming safe and effective procedure. It can be used as a bridge to surgery in patients who are critically ill or have several comorbidities.(17) Despite the widespread use of interventional radiology techniques, there has been a reluctance to apply these to spleen, due to concern for bleeding and difficulty in negotiation around colon and pleura.(18) Many recent studies have shown that percutaneous drainge is safe and splenectomy should be reserved for cases in which aspiration failed. Two important cautions must be observed. The coagulation profile must be normal and secondly remember that vascular lesions of spleen are difficult to recognise on imaging and percutaneous drainage always carries an inherent risk of catastrophic haemorrage. In the event of haemorrhage the patient will require embolization or emergency surgery for which facilities should be available.

\section{CONTRAINDICATIONS TO PERCUTANEOUS DRAINAGE:}

1. Uncontrollable coagulopathy.

2. Multiloculated or debris filled abscess.

3. Multiple small abscesses.

4. Diffuse ascites.

5. No safe route for drainage.

\section{RELATIVE CONTRAINDICATIONS:}

1. Splenic abscess secondary to contagious process in colon, pancreas, or stomach or a subdiaphragmaic abscess.

2. Ruptured abscess.

3. Phlegmon or a poorly localised lesion.

\section{COMPLICATIONS OF PERCUTANEOUS DRAINAGE:}

1. Bleeding.

2. Iatrogenic injuries to left kidney, colon, stomach, diaphragm, intestine.

3. Pleural puncture leading to pneumothorax or empyema.

SURGERY: Infection of splenic abscess can be brought under complete control by removal source ie splenectomy usually done by laprotomy although laparoscopic splenectomy is also being done successfully nowadays.(19) In many cases spleen has been destroyed completely by the overwhelming infective process and only open drainage of abscess by laparotomy can be done. In some cases spleen can be conserved by drainage of an abscess by surgery without removing the spleen i. e. a subtotal splenectomy.(20,21) Surgery remains the only alternative where the abscess is multifocal or multiseptate and other measures of antibiotic therapy or percutaneous drainage are unsuccessful. Surgery becomes mandatory when there is concommitant pathology like an associated bowel perforation. Complications of percutaneous drainage like bleeding or injuries to other organs have to be treated by surgery. Open surgery of splenectomy is more commonly done by midline or left subcostal incision. Thoracoabdominal incision is used when encountered with very large spleen with adhesions to the undersurface of diaphragm. 
Open drainage of splenic abscesses can be done by smaller incision or by extraperitoneal approach through the bed of 11th rib. Meticulous haemostasis and postoperative drainage are keys to success. Postoperatively the splenic bed can be continuously washed with saline by wide bore drainage tubes mechanically so as to remove the thick debris.

ADVANTAGES OF SURGERY: Surgery has the advantage of removal of infective focus completely. Faster recovery can be expected as the sepsis comes under control. Peritoneal cavity can be thouroughly cleaned and concommitant pathologies can be handled.

DISADVANTAGES OF SURGERY: Complications related to major operation and general anasthesia in a moribund immunocompromised patients are the major disadvantages Pulmonary complications in presence of severe abdominal sepsis can be disastrous. Atelectasis and bronchopneumonias are commonly seenwhich require interventions and ventilatory support and which can add to the morbidity and increase the mortality. Loss of spleen is a major disadvantage of surgical treatment. Conservation of spleenis highly recommended as it has definite immunological role. Postoperative surgical complications like intra peritoneal adhesions, scar hernias are inevitable sequeale of a laparotomy which are the major disadvantages.

SUMMARY: Splenic abscess is an uncommon clinical condition. Difficult to diagnose, the disease carries a high morbidity and mortality rate when it remains undiagnosed. It has a predisposition for the immunocompromised patients. Furthermore, with the advent of non-operative management of splenic trauma by embolization, potentially predisposing to formation of splenic abscess. The aim of this article was to review the clinical profile and methods of treatment. Different imaging modalities facilitate diagnosis, with the computed tomography being the cornerstone. Splenectomy remains the mainstay of the treatment, although encouraging results for percutaneous drainage have been reported. Splenic abscess in relevence to differential diagnosis of febrile illness should be suspected when we are confronted with patients with fever and pain in left subcostal region, especially, when they are under some form of immunosupression.

ACKNOWLEDGMENT: We the authors of this article report that there is no conflict of interests or funding to declare.

\section{REFERENCES:}

1. Constantin Fotiadis, Giagkos Lavranos, Pavlov Patapis, Gabriel Karatzas. Abscess of spleen: Report of three cases. World J Gastroenterol 2008 May 21; 14 (19): 3088-3091.

2. H. Sreekar, Vivek Saraf, Ashok C Pangi, H. Sreeharsha, Ravi Reddy, Goutam Kamat. Indian J Surg 2011 December; 73 (6): 398-402.

3. M Bernabeu-Wittel, JL Villanueva, J Pachon, A Alarcon, LF Lopez-Cortes, P Viciana et al. Etiology, Clinical features and Outcome of Splenic Microabscesses in HIV-Infected Patients with prolonged fever. Eur J Clin Microbiol Infect Dis 1999; 18: 324-329.

4. Chun C, Raff M, Contreas L. Changing clinical spectrum of splenic abscess: A multicenter study and review of literature. American Journal of Surgery 1987; 154: 27-34.

5. Jayssand R, Brasme L, Vernet-Garnier V, Deville JF, Atkas B et al. Splenic abscess complicating Salmonella Typhi infection. Eur J Clin Microbiol Infect Dis 2000; 19: 399-400. 
6. Choudhary R, Mahajan RK, Diwan A, Khan S, Singhal R, Chandel DS et al. Unusual presentation of enteric fever: Three cases of splenic and Liver Abscesses due to Salmonella Typhi and Salmonella Paratyphi A. Trop Gastroenterol 2003; 24: 198-199.

7. Caksen H, Oner AF, Arslan S, Koscoglu B, Harman M, Atas B et al. Splenic Abscess, pleural effusion and severe anemia caused by Salmonella Typhi. Kobe J Med Sci 2000; 46: 201-204.

8. Sharma S, Dey AB, Agarwal n, Nagarkar KM, Gujral S. Tuberculosis: A rare cause of splenic abscess. j Assoc Physicians India1999; 47: 740-741.

9. Reichel C, Theisen A, Rockstroh JK, Muller-Miny h, Spengler U, Saurbrunch T. Splenic abscess and abdominal tuberculosis in patients with AIDS. Z Gastroenterol 1996; 34: 494-496.

10. Bastounis E, Pikoulis E, Varelas P, Cirochiristos D, Aessopos A. Tuberculoma of the spleen: A rare but important clinical entity. Am Surg 1999; 65: 131-132.

11. Jonathan Figueroa, Marcel Tapia, Elisfat Arce, Micheal Wasung. Splenic Abscess due to Aspergillus. Calicut Medical Journal 2010; 8 (4): e6.

12. Loualidi A, Gerritsen M, Seelen JL, Kabel PJ, van Doorn LG. Splenic Abscess caused by Peptostreptococcus Species diagnosed with the aid of abdominal computerized tomography and treated with percutaneous drainage and antibiotics: A case repot Neth J Med 2001; 59: 280-285.

13. Cokca F, Yilmaz-Bozkurt G, Azap A, Memikoglu o, Tekeli F. Meningoencephalitis, pancytopenia, pulmonary insufficiencyand splenic abscess in a patient with Brucellosis. Sausdi Med J 2006; 27: 539-541.

14. Lee Ch, Leu HS, Hu Th, Liu JW. Splenic absces in Southern Taiwan. J MIcrobiol Immunol Infect 2004; 37: 39-44.

15. 15Chee Yung Ng, E Chuan Leong, Hong Chee Chang. Ten year series of Splenic Abscesses in a General hospital in Singapore. Annals of Academy of Medicine September 2008; Vol 37 No. 9: 749-752.

16. Quinn SF, Sonnenberg Evan, Cassola G, Wittich GR, Neff C. Interventional Radiology in Spleen. Radiology1986; 161: 289-291.

17. Zerem E, Bergsland J. Ultrasound guided percutaneous treatment ofsplenic abscess: The significance in treatment of critically ill patient. World J Gastroenterol 2006; 12: 7341-7345.

18. Lucey BC, Boland GW, Maher MM, Hahn PF, Gervais DA, Mueller PR. Percutaneous nonvascular splenic intervention: A 10 year review. AJR Am J Roentgenol 2002; 179: 1591-1596.

19. Carbonell AM, Kercher KW, Matthews BD, Joels CS, Sing RF, Heniford BT. Laparoscopic Splenectomy for splenic abscess. Surg Laparosc Endosc Percutan Tech 2004; 14: 289-291.

20. Nagem, Rachid G MSc, Petroinau, Andy Phd. Case note: Subtotal splenectomy for splenic abscess. Canadian Journal of Surgery 52. 4 (Aug 2009): E91-92.

21. C. S. Changchein, T. L. Tsai, S, S, Chiou, C. H. Kuo. Sonographic pattern of splenic abscess. AJR1992; 138: 259-262. 


\section{AUTHORS: \\ 1. Sanjay M. Datey \\ 2. Neelam Charles \\ 3. Himanshu Patidar \\ 4. Shibanshu Bandil \\ 5. Shaurya Bajpai \\ 6. Mohan Gadodia}

\section{PARTICULARS OF CONTRIBUTORS:}

1. Professor, Department of General Surgery, SAIMS, Indore.

2. Professor, Department of General Surgery, SAIMS, Indore.

3. Resident, Department of General Surgery, SAIMS, Indore.

\section{FINANCIAL OR OTHER} COMPETING INTERESTS: None
4. Resident, Department of General Surgery, SAIMS, Indore.

5. Resident, Department of General Surgery, SAIMS, Indore.

6. Resident, Department of General Surgery, SAIMS, Indore.

\section{NAME ADDRESS EMAIL ID OF THE CORRESPONDING AUTHOR:}

Dr. Sanjay M. Datey, \# 43, Chikitsak Nagar, Opposite Bombay Hospital, Indore-452010,

Madhya Pradesh.

E-mail: datesanjai@rediffmail.com

Date of Submission: 21/03/2015.

Date of Peer Review: 23/03/2015.

Date of Acceptance: 08/04/2015.

Date of Publishing: 20/04/2015. 